\title{
Research on Three-point Bending Mechanical Performance of Square Tube Structure Filled with Foam Aluminum
}

\author{
Kun YANG*, Yunjie SHA**, Tao YU*** \\ *College of Mechanical Engineering and Automation, Liaoning University of Technology, Jinzhou 121000, \\ China, E-mail: yangkunwh@163.com \\ **College of Mechanical Engineering and Automation, Liaoning University of Technology, Jinzhou 121000, \\ China, E-mail: 1243255774@qq.com \\ ***College of Mechanical Engineering and Automation, Liaoning University of Technology, Jinzhou 121000, \\ China,E-mail: jxyutao@lnut.edu.cn \\ crossref http://dx.doi.org/10.5755/j02.mech.28613
}

\section{Introduction}

Square tube is often subjected to bending load in practical application. The cross-section of the bearing tube will be deformation seriously under the bending load, which leads to the stress concentration and the bending resistance of the structure decreases sharply. And it is easy to bend collapse, while the bearing capacity of the tube reaches the limit value. So, it makes the bending resistance of the whole structure fail. The filling of foam aluminum into the tube can greatly reduce the deformation of the cross-section and increase the bearing capacity of the structure. The stability of the structure's bending resistance is improved.

The dynamic mechanical properties of foam aluminum epoxy composite sandwich beams were tested by threepoint bending impact test. The effects of different impact velocity and core thickness on impact load and energy absorption were analyzed. Compared with traditional sandwich beams and pure foam aluminum beams, the composite sandwich beams were better integrated in the parameters set up by Xin et al. [1] The effects of immersion corrosion on the mechanical properties of foam aluminum epoxy resin composites through three-point bending experiments, and found that immersion corrosion significantly reduced the bending mechanical properties of foam aluminum epoxy resin composites was studied by Yu et al. [2] In the case of the same size of matrix material and specimen, the influence of span on mechanical properties and failure modes of foam aluminum sandwich panels is confirmed by Yan et al. [3] By studying the bending problem of the double-modulus foam aluminum core sandwich beam, the transverse load acting on the double-modulus foam aluminum core sandwich beam has no influence on the neutral axis position of the double-modulus foam aluminum core sandwich beam under any boundary conditions, and the neutral axis position of the double-modulus foam aluminum core sandwich beam is determined, which has been proved by $\mathrm{Wu}$ et al. [4] Through experiments, the surface thickness, core height and angle direction were taken as variables respectively to analyze the factors affecting the flexural strength and failure mode of the sandwich beam. The theoretical strength of four failure modes was proposed by Yi-Ming Jen et al. [5] Through numerical and experimental studies on the bending performance of foam aluminum filled aluminum alloy tubes under the dynamic energy absorption, it can be observed that the energy absorption capacity increases at high strain rates, which is very helpful for anti-collision applications, and was found by Vignesh Sampathden et al. [6] Through the experiments of foam aluminum sandwich and fiber metal laminate on the basis of the new composite structure, compared with the traditional foam aluminum sandwich, the comprehensive performance of the new composite structure is improved, and the optimization parameters of the new foam aluminum composite structure with the best bending strength are obtained by Wang et al. [7] The quasi-static three point bending test of the foam aluminum sandwich beam at $25-500^{\circ} \mathrm{C}$ temperature range was used to investigate the quasi-static deformation and failure behavior of the sandwich beam. The failure mode diagram of the foam sandwich beam was extended to high temperature. The law of variation of initial failure mode diagram with temperature is found. The ultimate failure of foam aluminum sandwich beam is mainly caused by the shear of core layer by Li et al. [8] Through the three-point bending test of the fabricated metal sandwich structure, the initial failure load, peak load and failure mode were analyzed, and the initial failure mechanism was proved by L.L. Yan et al. [9] By comparing the theoretical calculation results and simulation analysis results with the experimental results, the mathematical model of the calculation formula of the bending moment and angle of the filled foam aluminum square tube structure was obtained, and the correctness of the theoretical analysis was proved.

\section{Experimental study}

\subsection{Sample preparation}

In this experiment, the material of the thin-walled square tube is Q235 low carbon steel. The section size is $30 \times 30 \mathrm{~mm}$ and the wall thickness is $1.2 \mathrm{~mm}$. The mechanical properties and stress-strain curves of tube wall materials are shown in Table 1 and Fig. 1, respectively.

\section{Table 1}

Material parameters of low carbon steel

\begin{tabular}{|c|c|}
\hline Parameter & Value \\
\hline Density $\rho, \mathrm{kg} \cdot \mathrm{m}^{-3}$ & 7860 \\
\hline Elastic modulus $E, \mathrm{MPa}$ & 206000 \\
\hline The tangent modulus $T, \mathrm{MPa}$ & 180 \\
\hline Poisson's ratio $\mu$ & 0.28 \\
\hline Yield strength $\sigma_{s,} \mathrm{MPa}$ & 235 \\
\hline
\end{tabular}

The closed cell foam aluminum used for filling the thin-walled square tube was produced by Shenyang Dongda 
Advanced Materials Development Co., Ltd by seepage casting method. The base material is ZL102 aluminum alloy with an average pore diameter of $3 \mathrm{~mm} 4 \mathrm{~mm}$ and a relative density of 0.22 . The mechanical properties and stress-strain curves are shown in Table 2 and Fig. 2, respectively.

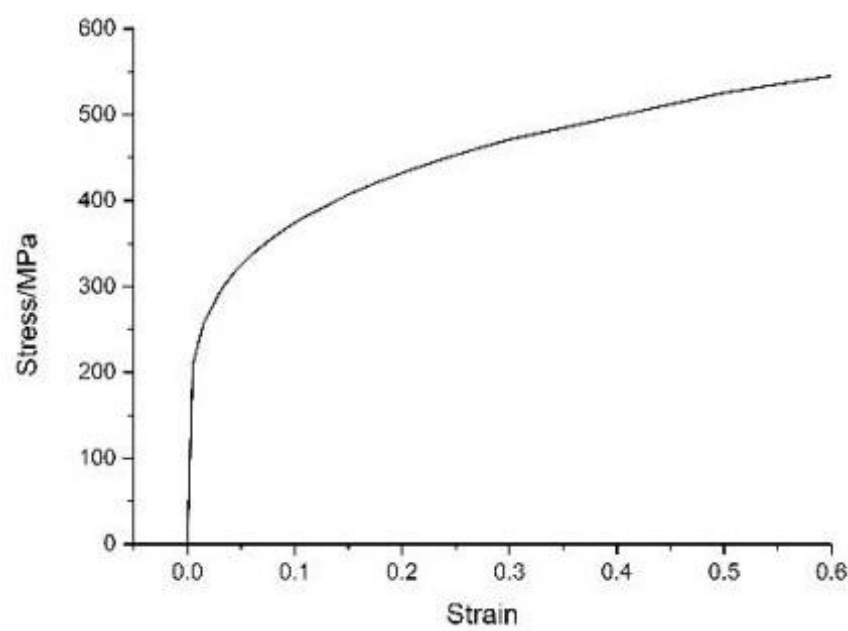

Fig. 1 Stress-strain of low carbon steel under strain rate 0.1

Table 2

Material parameters of closed-cell foam aluminum

\begin{tabular}{|c|c|}
\hline Parameter & Value \\
\hline Density $\rho /\left(\mathrm{kg} \cdot \mathrm{m}^{-3}\right)$ & 540 \\
\hline Elastic modulus $E / \mathrm{MPa}$ & 254 \\
\hline Poisson's ratio $\mu$ & 0.33 \\
\hline Yield strength $\sigma_{s} / \mathrm{MPa}$ & 8.1 \\
\hline
\end{tabular}

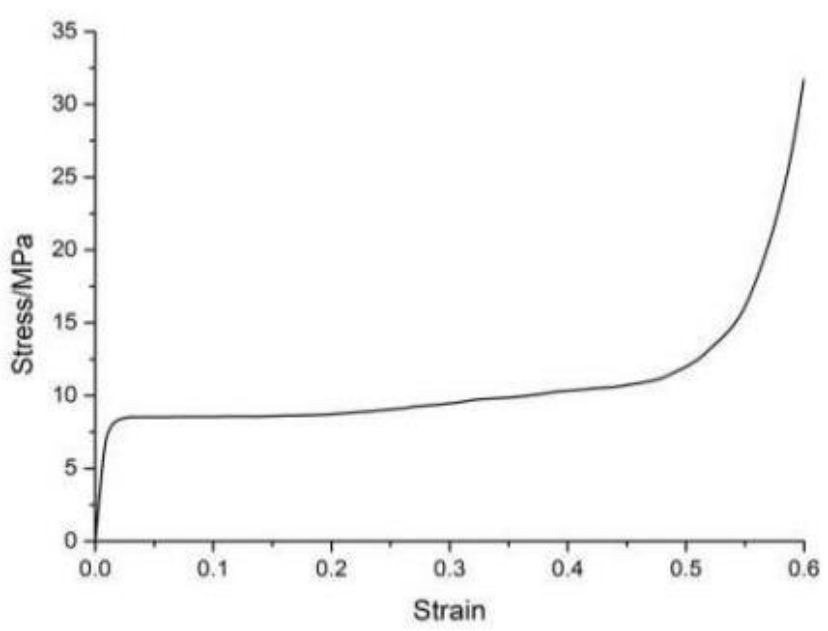

Fig. 2 Compression stress-strain of foam aluminum

Reference to YB/T 5349-2006 "bending mechanical properties of metal method" in the regulation of the relationship between the specimen length and span, and combined with the experimental conditions in the laboratory and the particularity of the three-point bending test of foam aluminum filled square tube, the span ratio of three-point bending test of foam aluminum filled square tube is determined to be $L_{s} / b=6.67$. So, the span of the specimen is $200 \mathrm{~mm}$, and the length is $240 \mathrm{~mm}$. The experimental schematic diagram is shown in Fig. 3. The diameter of the two supporting columns at the lower end and the cylinder punch at the upper end are both $20 \mathrm{~mm}$.

In order to analyze the influence of the bonding between the foam aluminum core and the tube wall on the bending deformation ability of the foam aluminum filled square tube, the foam aluminum was cut into rectangular shapes of $27 \times 27 \times 240 \mathrm{~mm}$ and $27.5 \times 27.5 \times 240 \mathrm{~mm}$. And two different sizes of the foam aluminum cores were filled into the empty square tube with the length of $240 \mathrm{~mm}$. The foam aluminum core with the cross-section size of $27 \times$ $27 \mathrm{~mm}$ was bonded to the tube wall by epoxy resin. There is clearance fit between the foam aluminum core with the tube wall in the other group, and the gap size is about $0.1 \mathrm{~mm}$. The final prepared specimens for the three-point bending test are shown in Fig. 4, such as empty tube (1-3), foam aluminum filled tube without glue ( 5 and 7 ), foam aluminum filled tube with glue ( 4 and 6 ).

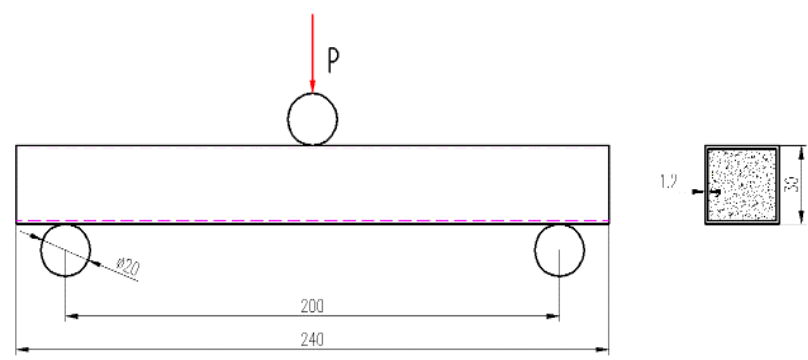

Fig. 3 Diagram of quasi-static three-point bending test

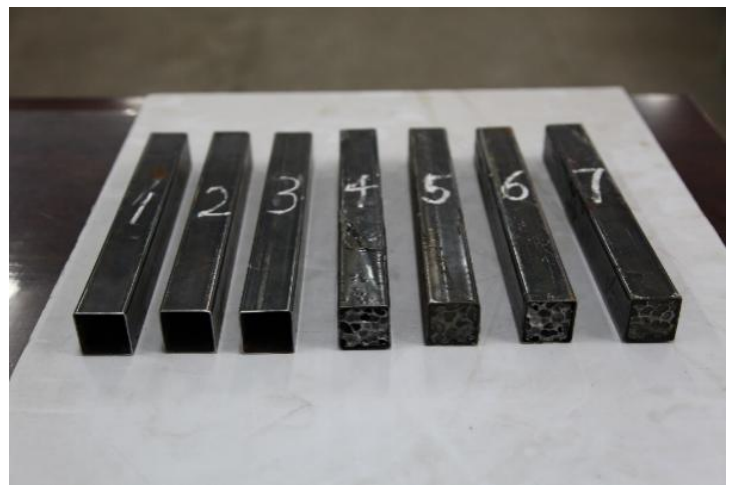

Fig. 4 Specimens for the three-point bending experiment

\subsection{Experimental equipment and methods}

The experiment is carried out on SANS-CMT5205 microcomputer controlled electronic universal experiment machine. Before the experiment, the specimen was freely placed on the two supported columns, the span of the specimen was set as $200 \mathrm{~mm}$. And the axis of the loading punch was aligned with the center line of the specimen, as shown in Fig. 5. The specimen can slide in the horizontal direction, but the displacement in the vertical direction is limited. Displacement loading method was adopted in the experiment, and the loading rate was $0.1 \mathrm{~mm} / \mathrm{s}$ (i.e. the strain rate was $10^{-2}$ ). During the experiment, the loading punch was gradually pressed down until the specimen was damaged or the loading displacement reached $65 \mathrm{~mm}$. The deformation and load-displacement curve of the specimen were observed in real time. After the end of the experiment, the load-displacement data were processed to obtain the bending momentangle curve of the square tube.

In order to compare and analyze the experimental results, the quasi-static three-point bending experiment of foam aluminum filled square tube and the square tube were carried out. Three samples of empty tube are tested, and two repetitions of foam aluminum filled square tube with and without glue are also tested, and the average value is taken as the experimental result. 


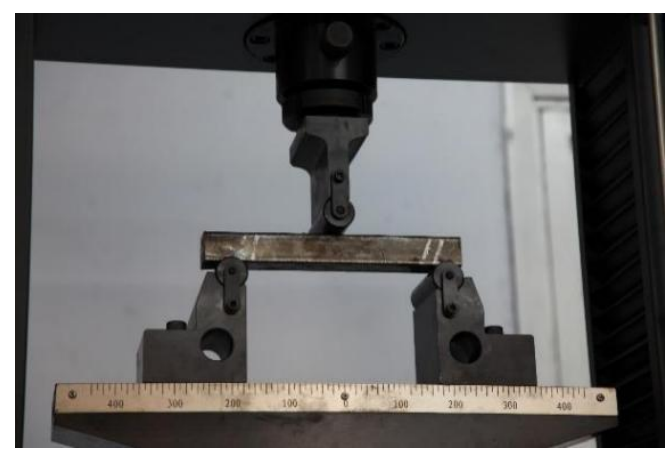

Fig. 5 Three-point bending experiment

\subsection{Experimental results and analysis}

In all cases, the two repetitions of the specimens show almost the same deformation behavior.

\subsubsection{Experimental results and analysis of empty square tube}

Fig. 6 shows the moment-angle curve of the threepoint bending experiment of the thin-walled square tube. It can be seen from the figure, in the initial stage of the experiment, the bending moment rapidly increases to the maximum value of $249.4 \mathrm{Nm}$. As the loading moment is greater than the plastic limit moment of the square tube structure, the tube walls on both sides of the square tube are extruded by the loading punch to produce plastic hinge. The bending deformation only occurs in this area, and the other parts only rotate. With the collapse of the local structure, the cross section of the square tube decreases sharply, and the bending moment decreases gradually with the increase of the angle of rotation. The bearing capacity of the structure decreases significantly. The bending moment value rises slightly, when the folds on both sides of the punch contact and squeeze each other. However, compared with the axial compression of square tube, the bearing capacity of bending deformation is much lower than that of compression deformation. Therefore, the bearing capacity and energy absorption of square tube under bending moment load or transverse load are not ideal. The final bending deformation results are shown in Fig. 7.

Through the observation of the experimental process, Wang et al. [10] found that the bending process of square tube can be divided into three stages:

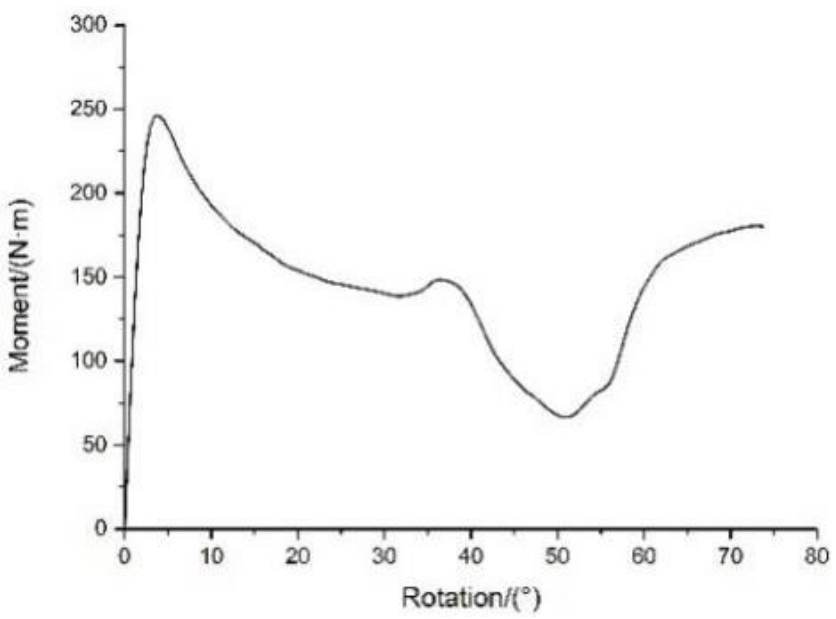

Fig. 6 Moment-rotation of square tube

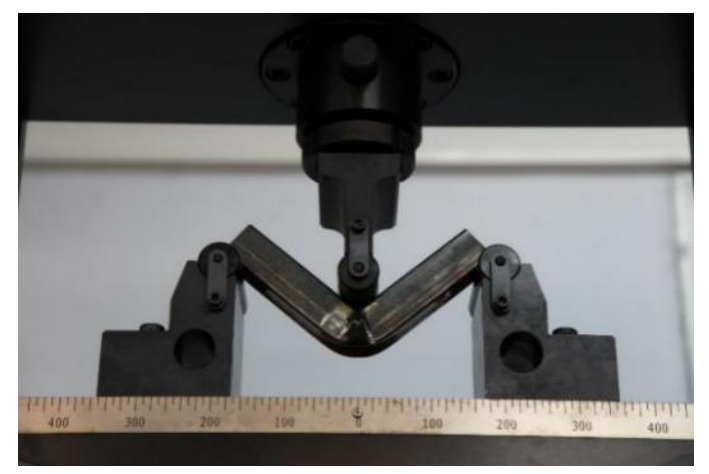

Fig. 7 Three-point bending experimental results of square tube

1. Initial elastic deformation stage: at this stage, the load of pressure is not greater than the yield strength of the square tube, and there is no plastic hinge movement deformation, that is no plastic deformation, only elastic deformation. At this time, the bulge begins to appear on the web of the square tube, and the bending moment increases rapidly to the maximum value. The bending angle ranges from $0^{\circ}$ to $5^{\circ}$, and has a linear relationship with the bending moment.

2. Bending deformation stage: this stage is the main stage of bending deformation. At this stage, the square tube structure has plastic bending deformation, which includes both bending around the static plastic hinge line and moving around the plastic hinge line. The bending moment decreases with the increase of the angle, and the bending angle ranges from $5^{\circ}$ to $30^{\circ}$.

3. The termination stage: with the ongoing of the loading, the moving plastic hinge lines of the left and right parts of the buckling side plate are squeezed after contacting each other, that leading to the moment rising. At this time, the original plastic hinge line stops moving and forms a new one. If the bending moment increases to exceed the tensile limit of the lower web plate of the square tube, the fracture occurs at this place. So, the whole bending deformation process is terminated.

\subsubsection{Experimental results and analysis of foam aluminum filled square tube}

Fig. 8 shows the moment-angle curves of the threepoint bending experiment of foam aluminum filled square tubes with bonded and unbonded epoxy resin.

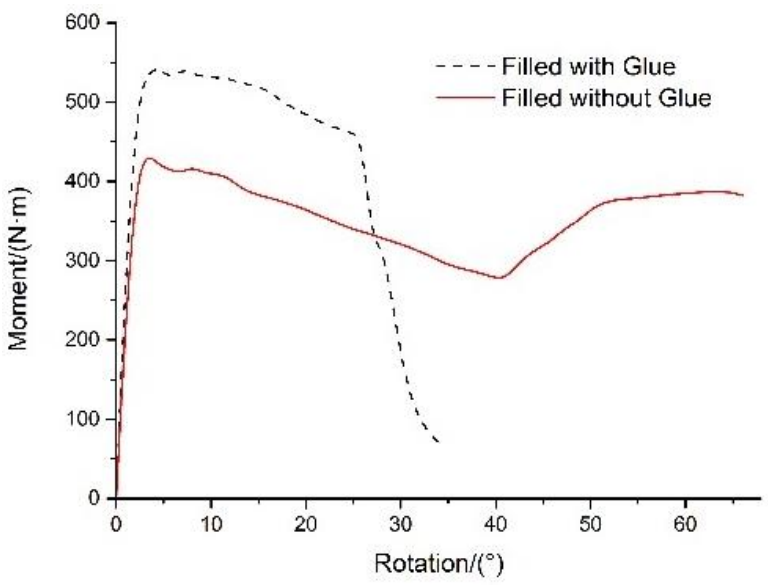

Fig. 8 Moment-rotation of foam aluminum filled tube with or without glue 
By comparing the bending moment-angle curve of the empty square tube, it can be found that the curve trend of the foam aluminum filled square tube shows similar deformation process, and the curve changes more smoothly. The bending process can also be divided into three stages: the elastic deformation stage, the bending deformation stage and the termination stage. However, the bending moment of foam aluminum filled square tube is greater than that of the empty square tube at each stage, especially the limit bending moment and its corresponding rotation angle. By comparing the bending moment-angle curves of the foam aluminum filled square tubes bonded and unbonded with epoxy resin, it is found that the limit bending moment of the foam aluminum filled square tubes bonded with epoxy resin is $542 \mathrm{Nm}$, which is higher than the ultimate bending moment of the unbonded tubes' $434 \mathrm{Nm}$. However, tearing failure occurs at a small angle, which makes the moment load decreased sharply. Fig. 9 shows the final deformation results of the bonded foam aluminum filled square tube.

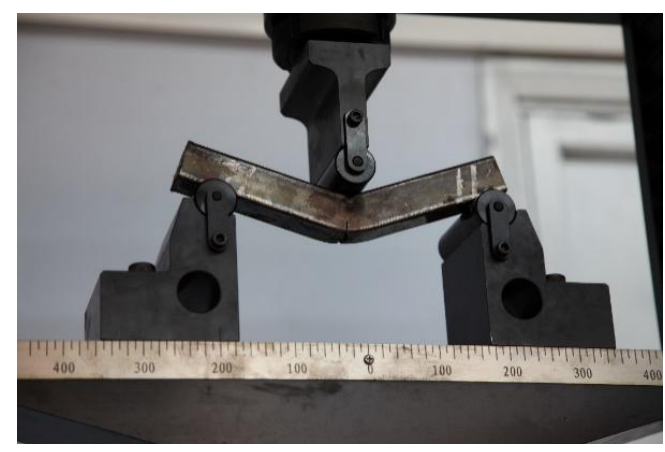

Fig. 9 Three-point bending experiment results of foam aluminum filled square tube with glue

The final deformation results of the three-point bending of the empty square tube and the foam aluminum filled square tube is shown in Fig. 10. Comparison with the bending process of the empty square tube, the bending deformation mode of the square tube is changed after filling the foam aluminum material. The filling foam aluminum core hinders the formation of complete folds in the compression side of the tube wall, which results in the reduction of the length of folds. It can also be seen that there is a tear failure on the lower surface of the foam aluminum filled square tube with adhesive under the compression area. This is mainly due to the connection between foam aluminum with tube wall by epoxy resin adhesive, which increases the stiffness of the foam aluminum core. It further limits the formation of the complete fold of the square tube. In the early stage of bending deformation, tensile tearing is easy to occur on the lower surface of the square tube, and the bending moment decreased sharply, which makes the whole filled structure failure.

From the experimental results, it can be found that the bonding mode of foam aluminum and tube wall further improves the bending stiffness and load capacity of the filling structure. But it also greatly limits the formation of the folds on the surface of the square tube, and causes the tensile stress of the lower surface too large, which leads to the failure of the filling structure at a small rotation angle. It is obvious that the adhesion between the tube wall and foam aluminum is not desirable for energy absorption since the bonding mode significantly reduces the total energy absorption capability and deformation uniformity of the structure.

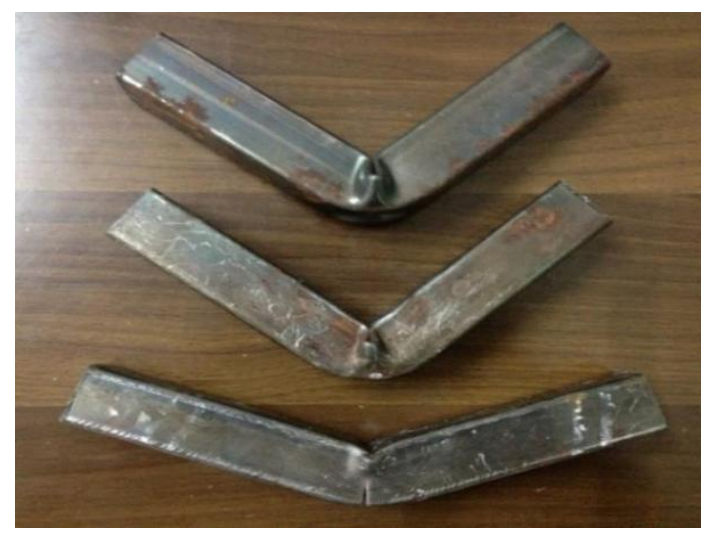

Fig. 10 Deformation mode of empty tube and tube with foam aluminum filler: from top to bottom: empty tube, filled without glue, filled with glue

\section{Quasi-static bending performance of foam aluminum filled square tube}

According to Wierzbicki's [11] super beam element model of square tube bending, the bearing capacity of foam aluminum filled square tube under static bending deformation and the relationship between bending moment and turning angle were analyzed theoretically at small turning angle. The bending deformation diagram of foam aluminum filled square tube is shown in Fig. 11. The cross-section size of the square tube is $b \times b$, the wall thickness of the tube is $t$, and the angle of the square tube is $\theta$ during bending. The maximum bending moment of the section of the filled square tube appears at the midpoint of the section. From the deformation results in the Fig. 11, it can be deduced that the relationship between the bending moment of the structure and the rotation angle is:

$$
\begin{aligned}
& M=\frac{P L}{4}, \\
& \theta=2 \arctan \frac{2 \delta}{L} .
\end{aligned}
$$

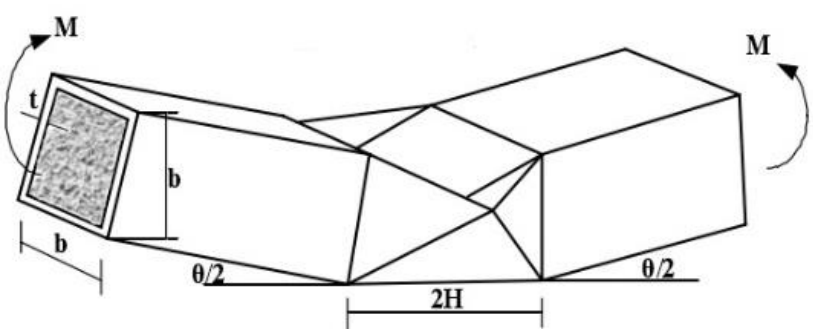

Fig. 11 Deformation of square tube with foam aluminum filler during three-point bending

\subsection{Loading capacity of foam aluminum filled square tube}

The stress analysis of the foam aluminum filled square tube structure shows that the composite structure will undergo elastic bending deformation in the initial elastic bending stage. At this time, due to the action of the punch load, the bending deformation of the tube is composed of two parts: bending moment deformation and shear force deformation. If $L / b \geq 4$, the influence of shear force can be ignored in the calculation, because the additional deformation 
generated by shear force will not exceed $5 \%$ of the maximum deflection. According to the parallel axis law of the moment of inertia of the section, the moment of inertia of the square tube in Fig. 12 can be obtained as follows:

$$
I_{\mathrm{f}}=\frac{b^{4}-(b-2 t)^{4}}{12} .
$$

The moment of inertia of foam aluminum core is:

$$
I_{\mathrm{c}}=\frac{(b-2 t)^{4}}{12} \text {. }
$$

It can be concluded that the equivalent bending stiffness $(E I)_{e q}$ of foam aluminum filled square tube structure is:

$$
(E I)_{e q}=\frac{b^{4}-(b-2 t)^{4}}{12} E_{f}+\frac{(b-2 t)^{4}}{12} E_{c} .
$$

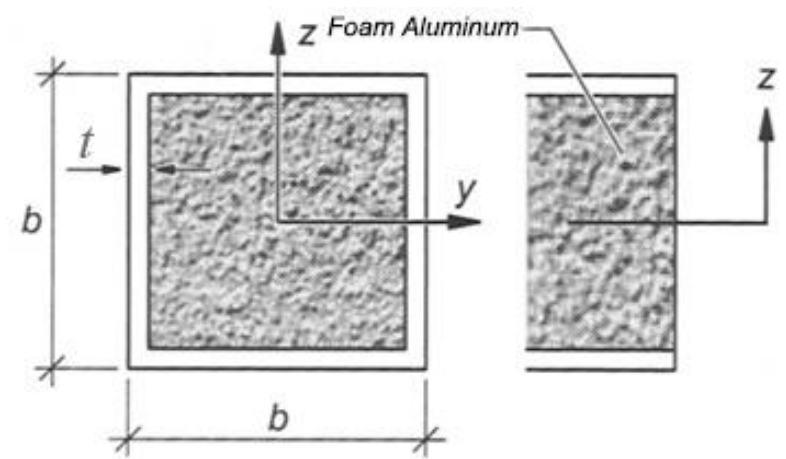

Fig. 12 Section of square tube with foam aluminum filler

In the initial elastic bending stage, the foam aluminum filled square tube has not occurred plastic deformation, and the interaction between the foam aluminum filled core and the tube wall can be ignored. So, the bending deflection of the foam aluminum filled square tube in the elastic bending stage is:

$$
\delta=\frac{P L^{3}}{48(E I)_{e q}} .
$$

By substituting Eqs. (1) and (2) into Eq. (6), in the case of small rotation angle of elastic bending, let $\tan \theta \approx \theta$ , then the relationship between bending moment and rotation angle is approximately linear:

$$
M \approx\left\{\left[b^{4}-(b-2 t)^{4}\right] E_{f}+(b-2 t)^{4} E_{c}\right\} \frac{\theta}{6 L} .
$$

The maximum bending strength of the square tube is controlled by the elastic buckling of the compression side of the thin-walled section. The critical stress $\sigma_{c r}$ on the compression side is expressed by Rhodes J. and Harvey J. M., [12] as:

$$
\sigma_{c r}=\frac{5.39 \pi E_{f}}{12\left(1-\mu^{2}\right)}\left(\frac{t}{b}\right)^{2} \text {. }
$$

Considering the strengthening effect of the tube wall material, the maximum bending moment of thin-walled square pipe can be approximated as:

$$
M_{f}=M_{p}-\left(M_{p}-M_{e}\right)\left(\frac{\sigma_{e}}{\sigma_{c r}}\right) \text {. }
$$

The parameters $\sigma_{e}, M_{p}$, and $M_{e}$ are defined as:

$$
\begin{aligned}
& \sigma_{e}=\sigma_{y}-\left(\sigma_{u}-\sigma_{y}\right) \frac{\sigma_{y}}{\sigma_{c r}}, \\
& M_{p}=\sigma_{y} \frac{b^{3}-(b-2 t)^{3}}{4}, \\
& M_{e}=\sigma_{y} \frac{b^{4}-(b-2 t)^{4}}{6 b} .
\end{aligned}
$$

After filling foam aluminum core into square tube, the bearing capacity of filled tube is improved, which is showed as the increase of limit bending moment and delay. The foam aluminum filling is equivalent to increasing the critical stress of the thin-walled tube. Through experiments, $\mathrm{Xu}$ et al. [13] found that the increased value $\Delta \sigma_{c r}$ of the critical stress $\sigma_{\text {cr }}$ can be expressed as:

$$
\begin{aligned}
\frac{\Delta \sigma_{c r}}{\sigma_{c r}} & =2 \pi\left(\frac{\rho_{f}}{\rho_{s}}\right)^{2.5}\left(\frac{t}{b}\right)\left(\frac{E_{f}}{\sigma_{y}}\right) \\
\sigma_{c r}^{\prime} & =\sigma_{c r}+\Delta \sigma_{c r} \text { is substituted into Eqs. (8) - }
\end{aligned}
$$

as the new critical stress value, and the maximum bending moment $M_{c f}$ of foam aluminum filled thin-walled square tube can be obtained. By substituting $M_{c f}$ into Eq. (7), the critical angle of the square tube filled with foam aluminum under the maximum moment can be obtained.

$$
\theta_{c f}=\frac{6 M_{c f} L}{\left[b^{4}-(b-2 t)^{4}\right] E_{f}+(b-2 t)^{4} E_{c}} .
$$

By substituting Eq. (14) into Eq. (7), the maximum bending moment of foam aluminum filled square tube can be obtained.

$$
M_{c f}=\left\{\left[b^{4}-(b-2 t)^{4}\right] E_{f}+(b-2 t)^{4} E_{c}\right\} \frac{\theta_{c f}}{6 L} .
$$

3.2. Quasi-static bending deformation of foam aluminum filled square tube

By observing the results of the three-point bending experiment, the bending moment of foam aluminum filled square tube can be divided into two parts: the bending moment of square tube and the bending moment of foam aluminum filled core.

Wierzbicki derived the moment-angle formula in the process of plastic bending deformation of square tube by 
using the super beam element model as follows:

$$
\begin{aligned}
& M(\theta)=2 P_{m} b\left(0.576+\frac{1}{2 \sqrt{\theta}}\right), \\
& P_{m}=2.76 \sigma_{0} b^{\frac{1}{3}} t^{\frac{5}{3}} .
\end{aligned}
$$

The equivalent stress $\sigma_{0}$ is approximated as:

$$
\sigma_{0}=\frac{\sigma_{u}+\sigma_{y}}{2}
$$

Then, the bending moment-rotation relationship of the thin-walled square tube can be expressed as:

$$
M(\theta)=\left\{\begin{array}{lr}
M_{f} & 0 \leq \theta<\theta_{\mathrm{c}} \\
2 P_{m} b\left(0.576+\frac{1}{2 \sqrt{\theta}}\right) & \theta>\theta_{\mathrm{c}}
\end{array} .\right.
$$

It should be pointed out that Equation (19) is only applicable to the case of bending angle less than $30^{\circ}$.

Through the observation of the experimental process, it is found that the collapse of the bending part of the square tube is prevented from filling of foam aluminum material. The bending resistance of the square tube is improved. Specifically, the maximum bending moment and critical turning angle of foam aluminum filled square tube are improved compared with those of square tube. The maximum bending moment $M_{u f}$ can be expressed as:

$$
M_{u f}=M_{f}+\Delta M
$$

$\Delta M$ is related to the relative density of foam aluminum and the size of structural section, and its expression is as follows:

$$
\Delta M=\Delta \sigma_{u} \cdot \frac{3}{2} t b^{2} \sigma_{y}
$$

$\Delta \sigma_{u}$ is the increment of the bending strength of the filled structure compared with the square tube, which can be expressed as:

$$
\Delta \sigma_{u}=0.34 \frac{\sigma_{f}}{\sigma_{y}}\left(\frac{\rho_{f}}{\rho_{s}}\right)^{1 / 2} .
$$

Substituting Eq. (22) into Eq. (21), we can get:

$$
\Delta M=0.51 t b^{2} \sigma_{f}\left(\frac{\rho_{f}}{\rho_{s}}\right)^{1 / 2} .
$$

Similarly, the critical bending angle of foam aluminum filled square tube can be expressed as:

$$
\theta_{c f}=\theta_{c}+\Delta \theta=\theta_{c}+3.98 \frac{\rho_{f}}{\rho_{s}} .
$$

Therefore, the relationship between bending moment and angle in the whole bending deformation process of foam aluminum filled square tube can be expressed as:

$$
M_{f}(\theta)=\left\{\begin{array}{lr}
M_{u f} & 0 \leq \theta<\theta_{c f} \\
P_{m} b\left(\frac{1}{\sqrt{\theta}}-\frac{1}{\sqrt{\theta_{c f}}}\right)+M_{u f} \quad & \theta \geq \theta_{c f}
\end{array} .\right.
$$

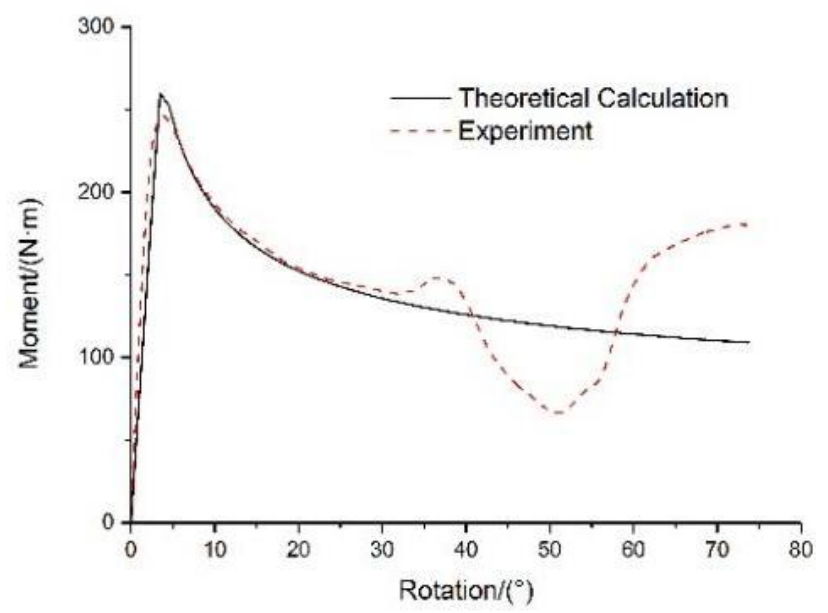

Fig. 13 Comparison on theoretical and experiment threepoint bending results of empty square tube

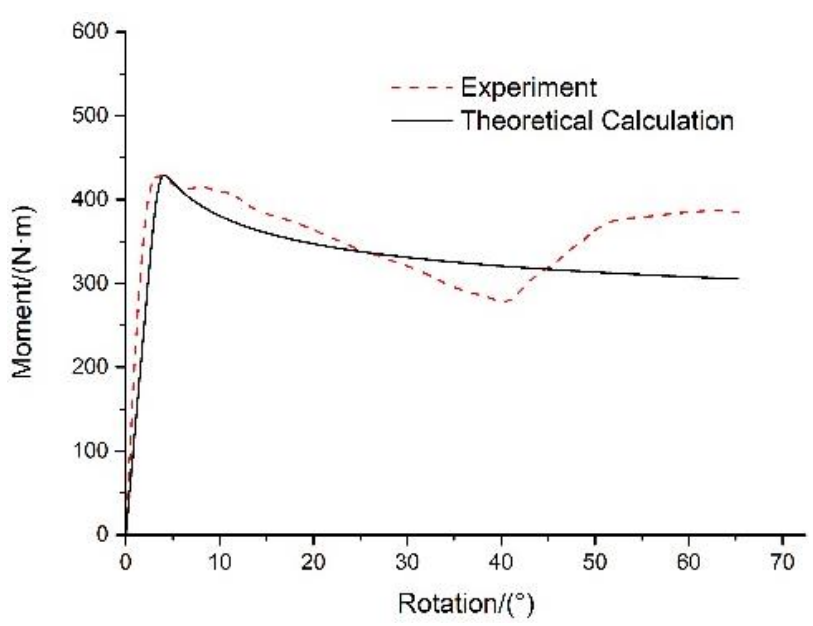

Fig. 14 Comparison on theoretical and experiment threepoint bending results of square tube with foam aluminum filler

As shown in Fig. 13 and Fig. 14, the theoretical calculation results are compared with the experimental results of the three-point bending of square tube and foam aluminum filled square tube respectively. It can be seen from the figure that the theoretical calculated results of the square tube are in good agreement with the experimental values at the initial stage of bending. However, the theoretical values of the filled tube are smaller than the experimental values. It may be that the theoretical analysis does not fully take account of the influence of foam aluminum filling in the structure, nor does it account for the stress strengthening stage of some aluminum foam materials during bending deformation.

At large rotating angles, the theoretical values of square tube and filled tube have a large deviation from the experimental values, which indicates that the theoretical 
analysis is only applicable to the bending of small rotating angles.

\section{Finite element numerical simulation study}

In order to further study, the accuracy of theoretical analysis, the three-point bending deformation of foam aluminum filled square tube under quasi-static and dynamic conditions is simulated by using HyperMesh and LSDYNA. And the simulation results are compared with the experimental results.

\subsection{Model building and meshing}

2D shell 163 thin shell element is used in the square tube, the thickness of the element is $1.2 \mathrm{~mm}$, the shape of the element is quadrilateral, and the mesh size is $2 \mathrm{~mm}$. The foam aluminum core is divided by 3D Solid164 element, the element shape is hexahedral, and the mesh size is $2 \mathrm{~mm}$. The supporting columns and the loading punch are divided by shell element, and the grid is $4 \mathrm{~mm}$. The bending model of foam aluminum filled square tube is divided into 23,716 solid elements and 7,200 shell elements. The three-point bending finite element model is shown in Fig. 15.

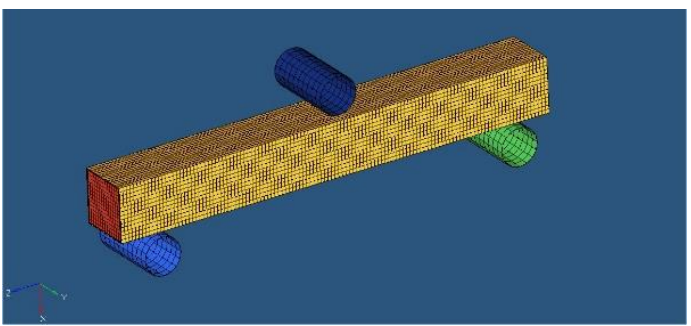

Fig. 15 Finite element model of three-point bending

\subsection{Analysis of bending simulation results}

\subsubsection{Quasi-static simulation results and analysis}

As shown in Fig. 16, the experimental data of the quasi-static three-point bending of the empty square tube are compared with the simulation results.

By comparing the two curves, it is found that the simulation results have a similar trend with the experimental data at a small rotate angle, while the deviation is large at a large rotate angle. The simulated limit bending moment of the square tube is $290 \mathrm{Nm}$, which is slightly higher than the 249.4 Nm measured by experiment.

The simulation results of the quasi-static threepoint bending of the foam aluminum filled square tube are shown in Fig. 17. Comparing with the experimental data, it is found that the moment-rotation curve obtained by the simulation is close to the experimental data without bonding in the small angle, and the difference is larger when the angle is greater than $35^{\circ}$. The main reason is that the folds generated by the tube wall surround the punch well in the analysis, and the folds on both sides of the punch do not contact with each other in the large angle bending, which makes the simulated load curve very smooth. The simulated limit moment of quasi-static bending of foam aluminum filled square tube is $452 \mathrm{Nm}$, which is slightly higher than $434 \mathrm{Nm}$ measured by experiment.

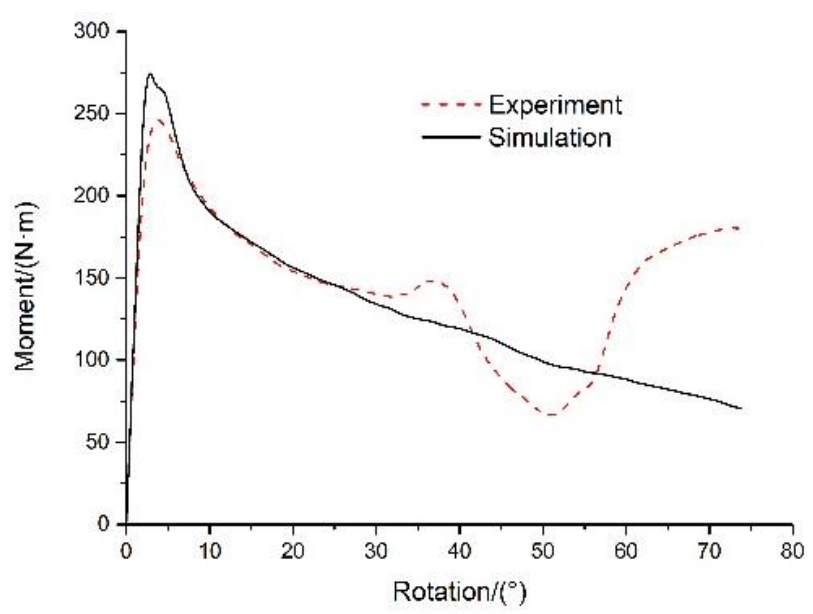

Fig. 16 Three-point static bending moment-rotation of empty square tube

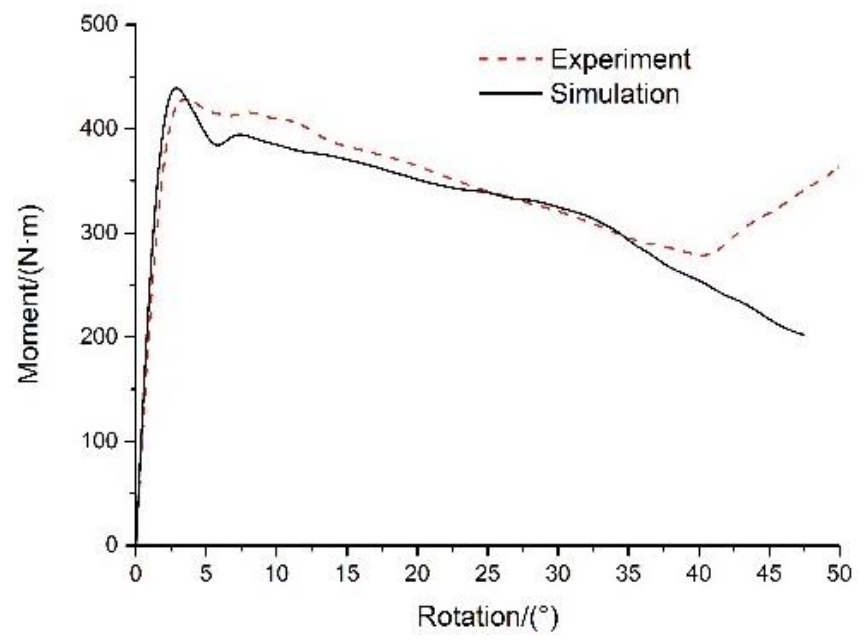

Fig. 17 Three-point static bending moment-rotation of square tube with foam aluminum filler

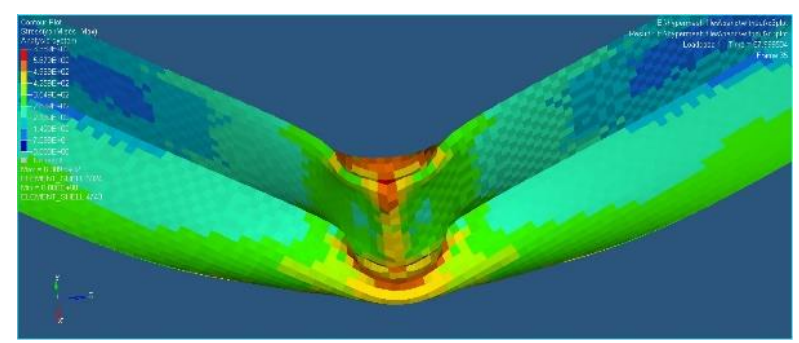

Fig. 18 Three-point static bending result of square tube

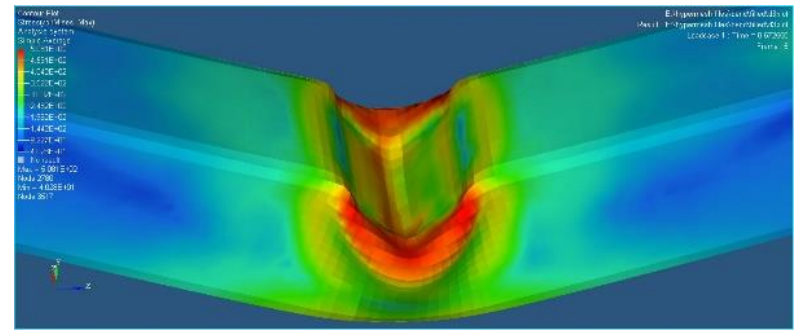

Fig. 19 Three-point static bending result of square tube with foam aluminum filler

Figs. 18 and 19 are the final deformation results of the quasi-static bending deformation of the square tube and the foam aluminum filled square tube respectively. Combined with the experimental results of three-point bending, it can be seen that the finite element model can well simulate 
the deformation results at small rotation angles, but the final deformation results are quite different from the experimental results, and there is no contact and extrusion of folds.

\subsubsection{Simulation results and analysis of dynamic bending}

The simulation analysis results of dynamic threepoint bending of square tube is shown in Fig. 20. It can be seen from Fig. 20 that the limit bending moment of the tube under the impact load is greatly increased compared with that under the of under quasi-static load. The limit bending moment of the tube under the impact load is $788 \mathrm{Nm}$, which is 2.7 times of the quasi-static condition. This is because the yield stress of low carbon steel material increases obviously under high strain rate loading conditions. While the rotation angle of the square tube increases to about $80^{\circ}$, the load decreases rapidly and the deformation tends to stop. It indicates that the impact energy is completely absorbed.

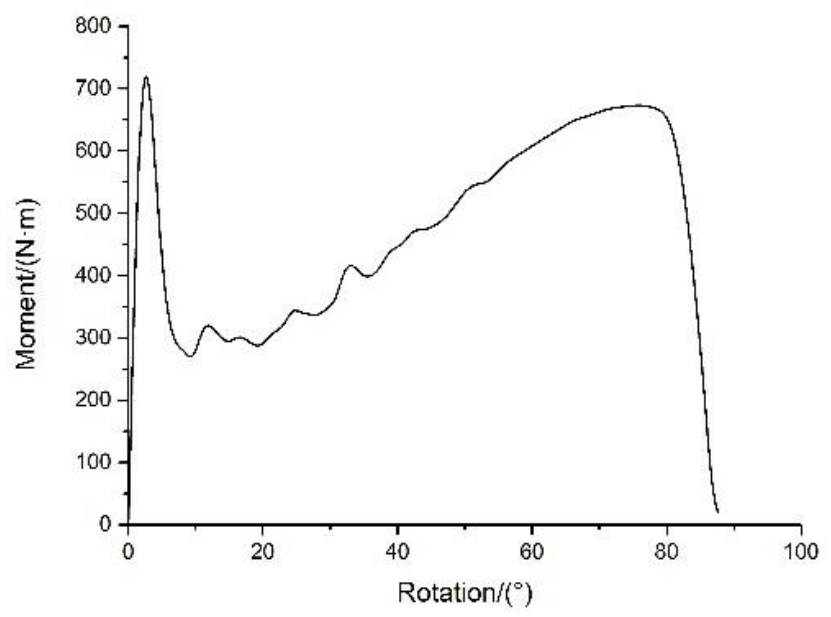

Fig. 20 Three-point dynamic bending moment-rotation of square tube

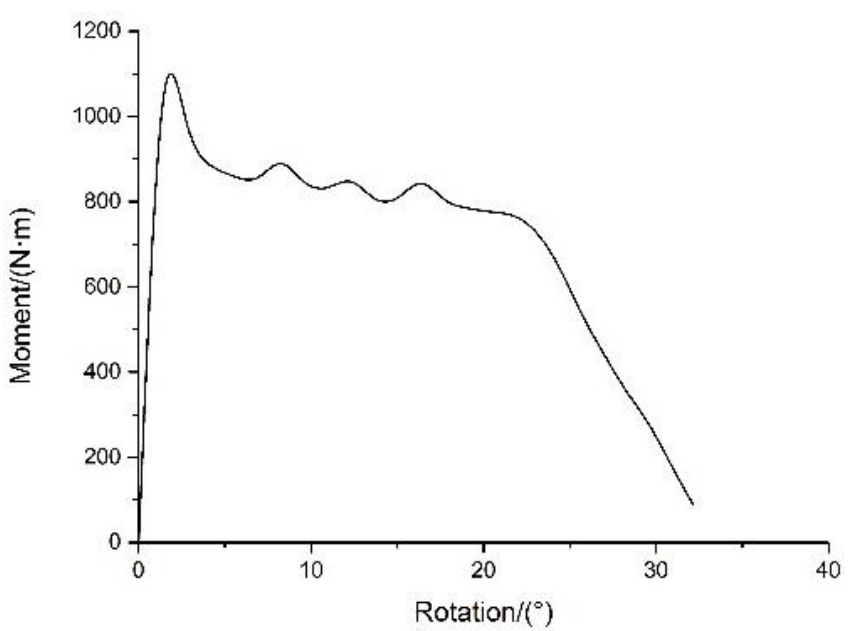

Fig. 21 Three-point dynamic bending moment-rotation curve of square tube with foam aluminum filler

The simulation analysis results of dynamic threepoint bending of foam aluminum filled square tube is shown in Fig. 21. As can be seen from Fig. 21, the limit bending moment of foam aluminum filled square tube under impact loading is much higher than that of under quasi-static load. The limit bending moment of foam aluminum filled square tube in impact process is $1289 \mathrm{Nm}$, which is 2.9 times of the quasi-static bending moment $434 \mathrm{Nm}$. While the angle of the square tube increases to about $33^{\circ}$, the deformation tends to stop. By comparison with Fig. 20, the bending angle of the foam aluminum filled square tube is significantly lower than that of the square tube under the same impact loading conditions, and the limit bending moment of the foam aluminum filled square tube is also higher than that of the square tube. It indicates that the bending bearing capacity and energy absorption performance of the square tube have been greatly improved after the filled with foam aluminum.

\section{Conclusions}

In this paper, the three-point bending properties of square tube and foam aluminum filled square tube are studied experimentally by means of quasi-static mechanical loading experiment respectively. The moment-angle curves of the two types of square tube are obtained. The deformation mode, bearing capacity and energy absorption efficiency of two types of square tube are discussed by analyzing the obtained moment-angle curve and observing the experimental deformation results. The results show that the filling of foam aluminum can improve the bearing capacity and energy absorption performance of the square tube. Under the bending load, the deformation of the bearing section is greatly reduced, which increases the bearing capacity and the bending resistance stability of the structure. The bonding mode of foam aluminum to the tube wall further improves the bending stiffness and bearing capacity of the filled structure, but leads to the failure of the filled structure at a small turning angle. Therefore, it is obvious that the adhesion between the tube wall and foam aluminum is not desirable for energy absorption.

Based on the results of experimental and theoretical analysis, the static bending deformation behavior of the square tube filled with foam aluminum is deeply analyzed and studied. The mathematical model of the moment rotation for the square tube filled with foam aluminum under the static bending load is established. Furthermore, the threepoint bending deformation of the square tube and ones filled with foam aluminum under quasi-static and impact loading is simulated. The simulation results show that the finite element method is feasible to analyze the bending deformation behavior of the square tube filled with foam aluminum under quasi-static loading. Comparing with the simulation, theoretical and experimental results of the three-point bending deformation of the square tube and ones filled with foam aluminum, the error among each other is small in the case of small corner bending. So, it provides a theoretical basis for the design of the square tube filled with foam aluminum. Due to the limitation of experimental conditions, the threepoint bending performance of square tube filled with foam aluminum under dynamic loading is simulated and analyzed by finite element method. Therefore, the theoretical model under dynamic loading needs further study.

\section{References}

1. Xin, Y. J.; Yan, H. M.; Cheng, S. L.; Xiao, B.; Yu, W.; Li, H. J. 2020. Experimental study on dynamic three-point bending of aluminum foam-epoxy composite sandwich beam, Journal of Yanshan University 44(1): 87-94.

https://doi.org/CNKI:SUN:DBZX.0.2020-01-013. 
2. Yu, W.; Xue, H. L. 2017. Effect of immersion corrosion on flexure properties of aluminum foamed epoxy resin composites, Materials Review 31(22): 111-115+ 141. https://doi.org/10.11896/j.issn.1005-023X.2017.022. 022.

3. Yan, C.; Song, X. D.; Jing, C. H.; Feng, S. 2017. The influence of span on the mechanical properties and deformation modes of the three-point bending of aluminum foam sandwich. (eds.) China mechanics conference -2017 and the celebration of the 60th anniversary conference of the China Society of mechanics. Available from: http://cpfd.cnki.com.cn/Article/CPFDTOTALAGLU201708002036.htm.

4. Wu, X.; Yang, L. J. 2015. Calculation and analysis of bending of double modulus aluminum foam sandwich beams, Journal of applied mechanics 32(06): 922927+1097-1098. https://doi.org/10.11776/cjam.32.06. B131.

5. Jen, Y. M.; Tang, Z. H.2020. Static bending strength assessment of sandwich panels with glass/polypropylene faces and aluminum foam cores, Modern Physics Letters B34(7n9): 5. https://doi.org/10.1142/S0217984920400163.

6. Sampath, V.; Rao, C. L.; Reddy, S. 2017. Energy absorption of foam filled aluminum tubes under dynamic bending, Procedia Manufacturing 7: 225-233. https://doi.org/10.1016/j.promfg.2016.12.054.

7. Wang, Z. N.; Chen, X.; Li, A.; Li, Y. X.; Zhang, H. W.; Liu, Y. 2016. Three-point bending performance of a new aluminum foam composite structure, Transactions of Nonferrous Metals Society of China (02) 359-368. https://doi.org/10.1016/S1003-6326(16)64088-8.

8. Li, Z. B.; Lu, F. Y. 2015. The three-point bending deformation and failure mechanism of sandwich beams with foam aluminum at different temperatures. The office of the China Institute of mechanics. (eds.) Abstract of -2015 thesis of China mechanics conference. Available from: http://cpfd.cnki.com.cn/Article/CPFDTOTAL-AGLU201508002625.htm.

9. Yan, L. L.; Han, B.; Yu, B.; Chen, C. Q.; Zhang, Q. C.; Lu T. J. 2014. Three-point bending of sandwich beams with aluminum foam-filled corrugated cores, Materials \& Design 60: 510-519.

https://doi.org/10.1016/j.matdes.2014.04.014.

10. Wang, S. L.; Feng, Y.; Xu, Yi.; Wang, X. B.; Shen, J. 2007. Study on longitudinal and transverse compressive mechanical properties of laminated aluminum foam tubes. Journal of materials heat treatment 28(1): 10-15. https://doi.org/10.3969/j.issn.1009-6264.2007.01. 003.

11. Wierzbiekl, T.; Recke, L.; Abramowiez, W.; Gholami, T. 1994. Stress profiles in the thin-walled prismatic columns subjected to crush loading- II , Bending. Computers and Structures 51(6): 625-641.
https://doi.org/10.1016/S0045-7949(05)80001-X.

12. Rhodes, J.; Harvey, J. M. 1971. Design of thin-walled beams, Conference Exp Stress Analysis in Design, London 159-167, retrieved from:

http://trid.trb.org/view/103651.

13. Xu, K.; K, D. P.; Wang, E. H.; Yu, J. L. 2005. Static bending collapse behavior of thin-walled square aluminum tubes filled with aluminum foam, Journal of Solid Mechanics (03): 261-266. https://doi.org/10.19636/j.cnki.cjsm42-250/o3.2005.03. 003.

K. Yang, Y. Sha, T. Yu

\section{RESEARCH ON THREE-POINT BENDING MECHANICAL PERFORMANCE OF SQUARE TUBE STRUCTURE FILLED WITH FOAM ALUMINUM}

\section{$\mathrm{S} \mathrm{u} \mathrm{m} \mathrm{m} \mathrm{a} \mathrm{r} \mathrm{y}$}

In this paper, the quasi-static three-point bending experiments are carried out to study the deformation behavior of square tube and square tube filled with foam aluminum. The difference of bending deformation mode, loading characteristics and energy absorption efficiency between tube and foam aluminum filled tube is compared. And the influence of adhesive between the foam aluminum core and the tube wall on the bending deformation of square tube filled with foam aluminum is analyzed. Based on the bending super beam element model of tube structure, the relationship between the moment and rotation of square tube filled with foam aluminum under transverse static loading is analyzed. And the formula for calculating the moment and rotation angle of square tube filled with foam aluminum at three-point bending is obtained. In order to compare the simulation results, theoretical calculation results and experimental results of quasi-static bending, the three-point bending deformation of square tube and filled with foam aluminum under quasi-static and impact loading is simulated by finite element method. The results show that the filling of foam aluminum can improve the bearing capacity and energy absorption performance of the square tube structure. Under the bending load, the deformation degree of the bearing section is greatly reduced, which increases the bearing capacity of the structure and increases the stability of its bending resistance.

Keywords: foam aluminum; filling structure; square tube; bending resistance; three-point bending.

Received March 07, 2021 Accepted December 07, 2021

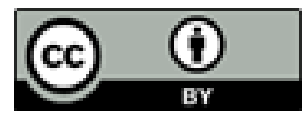

This article is an Open Access article distributed under the terms and conditions of the Creative Commons Attribution 4.0 (CC BY 4.0) License (http://creativecommons.org/licenses/by/4.0/). 